\title{
On Computing Some Extremal Periodic Positive-Definite Functions
}

\author{
By R. L. Page*
}

\begin{abstract}
This paper is concerned with maximizing the square integral over certain classes of periodic positive-definite functions. The question arose at Jet Propulsion Laboratory in connection with maximizing the average power of the received signal in radar exploration of the planets. We present computational evidence that the maximizing function exists and, in most but not all cases, is unique. Upper and lower bounds for the maximum square integral are computed, and formulas are conjectured for the maximizing functions in several cases.
\end{abstract}

1. Introduction. Let $\mathfrak{C}_{\delta}$ be the set of periodic, real-valued functions on the real line which are continuous, have period $2 \pi$ and satisfy

(1.1) $\Lambda(x)$ is positive definite; i.e., $\Lambda(x)=\sum_{n=0}^{\infty} p_{n} \cos n x$ where $\forall n, p_{n} \geqq 0$;

(1.2) $\Lambda(x)=0, \delta \leqq|x| \leqq \pi$

(1.3) $\Lambda(0)=1$.

Let $D_{\delta}=\max \left\{\int_{-\delta}^{\delta} \Lambda(x)^{2} d x: \Lambda \in \mathcal{C}_{\delta}\right\}$.

It is easy to show, by integrating over the domain $0 \leqq x \leqq \delta$ the inequality $\operatorname{det}\left\|\Lambda\left(x_{i}-x_{j}\right)\right\| \geqq 0$ with $x_{1}=0, x_{2}=x, x_{3}=\delta$, that $D_{\delta} \leqq \delta$. It is also true that $D_{\delta} \geqq \frac{2}{3} \delta$. One can see this by computing the square integral of the $\mathcal{C}_{\delta}$-function

$$
\Lambda(x)=(1-|x \bmod (-\pi, \pi]| / \delta)^{+} .
$$

A better lower bound is computed in a paper [3] by Garsia, Rodemich and Rumsey, in which the corresponding problem for nonperiodic functions on the real line is solved. They show that there is a function $\Lambda$ maximizing $\int_{-\delta}^{\delta}|\Lambda(x)|^{2} d x$ where the maximum ranges over the class $\mathcal{F}_{\delta}$ of continuous, positive-definite functions on the real line which vanish outside the interval $[-\delta, \delta]$ and take the value one at zero. Of course, for any value of $\delta \leqq \pi$, the class $\mathcal{F}_{\delta}$ can be embedded in $\mathcal{C}_{\delta}$ merely by constructing a periodic version of each function in $\mathcal{F}_{\delta}$. For any function $\Lambda$ in $\mathcal{F}_{\delta}$, the Fourier integral $\int_{-\delta}^{\delta} \Lambda(x) e^{-i x u} d x$ is nonnegative for all real values of $u$. This means that the corresponding periodic function $\Lambda^{*}(x)=\Lambda(x \bmod (-\pi, \pi])$ must be in $\mathfrak{C}_{\delta}$, since, in $\mathfrak{C}_{\delta}$, we only require that the Fourier integral be nonnegative for integer values of $u$. Therefore, the maximum square integral in the class $\mathcal{F}_{\delta}$, which Garsia,

Received July 24, 1972.

AMS (MOS) subject classifications (1970). Primary 42A88; Secondary 05-04, 65K05, 02E10, $85 \mathrm{~A} 45$.

Key words and phrases. Positive definite functions, maximization of convex functions, linear programming.

* This research was supported in part by the Air Force Office of Scientific Research, under contract no. 1322-67 and appeared as a part of the author's thesis at the University of California, San Diego, Department of Mathematics. 
et al. [3, p. 833] showed to be $.686981293033114600949413 \ldots \times \delta$, must be a lower bound on $D_{\delta}$. One would like to compute better bounds for $D_{\delta}$ and, perhaps, find a formula for the maximizing function, if one exists.

As stated, the problem is computationally intractable. A feasible computational approach is to consider an analogous class of discrete functions.

Given two integers $N$ and $\Delta(\Delta \leqq N / 2)$, let $Q(N, \Delta)$ be the set of periodic (period $2 \pi$ ) real-valued functions, $a$, defined on the $N$ th roots of unity, that is the set $\{2 \pi k / N: k=0, \pm 1, \pm 2, \cdots\}$, satisfying

(1.4) $a$ is positive-definite,

(1.5) $a(x)=0$ for $2 \pi(\Delta+1) / N \leqq|x| \leqq \pi$,

(1.6) $a(0)=1$.

Let

$$
B(N, \Delta)=\max \left\{(2 \pi / N) \sum a(2 \pi k / N)^{2}: a \in Q(N, \Delta)\right\} .
$$

The sum here, and elsewhere in this paper, is taken over any set of $N$ consecutive values of the integer $k$. Any such set gives the same sum because of the periodicity of the functions involved. We use the normalization $2 \pi / N$ here to make the measure of the roots of unity group the same as the measure of the circle group over which the class $\mathcal{C}_{\delta}$ is defined. Thus, $B(N, \Delta)$ is in units comparable to those of $D_{\delta}$.

If $\Lambda \in \mathfrak{C}_{\delta}$, then the $Q(N, \Delta)$-function produced by sampling $\Lambda$ at the mesh points $2 \pi k / N, k=0, \pm 1, \pm 2, \cdots$, must be in $Q(N, \Delta)$ as long as $\delta \leqq 2 \pi(\Delta+1) / N$. Furthermore, it can be shown, using a discrete version of the Poisson summation formula $[6$, p. 68], that

$$
(2 \pi / N) \sum \Lambda(2 \pi k / N)^{2} \geqq \int_{-\delta}^{\delta} \Lambda(x)^{2} d x .
$$

Therefore, $D_{\delta} \leqq B(N, \Delta)$ whenever $\delta \leqq 2 \pi(\Delta+1) / N$.

Conversely, given any function $a \in Q(N, \Delta)$, one can produce a function $\Lambda \in \mathfrak{e}_{\delta}$, $\delta=2 \pi(\Delta+1) / N$, by taking $\Lambda(2 \pi k / N)=a(2 \pi k / N)$ for all integers $k$, and linear in between those mesh points. That is, let

$$
\Lambda(x)=(1-\lambda) a(2 \pi k / N)+\lambda a(2 \pi(k+1) / N),
$$

where

$$
2 \pi k / N \leqq x \leqq 2 \pi(k+1) / N \text { and } \lambda=N(x-2 \pi k / N) / 2 \pi .
$$

A straightforward integration shows that the Fourier coefficients of $\Lambda$ are positive and that

$$
\begin{array}{rl}
\int_{-\delta}^{\delta} \Lambda(x)^{2} & d x \\
& =(2 \pi / N)\left[\frac{2}{3} \sum a(2 \pi k / N)^{2}+\frac{1}{3} \sum a(2 \pi(k-1) / N) a(2 \pi k / N)\right] .
\end{array}
$$

If we let $\operatorname{PL}(N, \Delta)$ denote the class of functions in $\mathcal{C}_{2 \pi(\Delta+1) / N}$ which are linear between the points $\{2 \pi k / N: k=0, \pm 1, \pm 2, \cdots\}$, and let $Q(N, \Delta)$ denote the maximal square integral in that class, then $D_{\delta} \geqq Q Q(N, \Delta)$ whenever $\delta \geqq 2 \pi(\Delta+1) / N$. Thus, we have upper and lower bounds on $D_{\delta}$ which depend on functions determined by a finite set of numbers. The maximal functions in the classes $Q(N, \Delta)$ and $\mathcal{P}(N, \Delta)$ can be computed using an algorithm described in Section 2. 
2. Computing the Maximal Functions in $Q(N, \Delta)$ and $\mathcal{Q}(N, \Delta)$. The problem of finding the maximal function in $Q(N, \Delta)$ may be stated as follows:

$$
\text { maximize } \sum a(2 \pi k / N)^{2}
$$

subject to the constraints (1.4), (1.5), (1.6) of Section 1.

Let $\left\{p_{n}\right\}$ be the Fourier transform of $a$; that is,

$$
p_{n}=(2 \pi / N) \sum a(2 \pi k / N) \exp (-2 \pi i k n / N) .
$$

Then, the requirement that the function $a$ be real-valued is equivalent to the requirement that $p_{n}=p_{-n}$ for all integers $n$. Furthermore, by the Fourier inversion formula, conditions (1.4), (1.5), and (1.6) of Section 1 are equivalent to the following constraints on the sequence $p_{n}$ :

$$
\begin{gathered}
p_{n} \geqq 0 \quad \text { for all integers } n, \\
(1 / 2 \pi) \sum_{n=0}^{N-1} p_{n} \exp (2 \pi i n k / N)=0, \quad-\Delta \leqq k \leqq \Delta, \\
(1 / 2 \pi) \sum_{n=0}^{N-1} p_{n}=1 .
\end{gathered}
$$

Using Parseval's identity and the symmetry of $\left\{p_{n}\right\}$, we can restate the problem of finding the maximal function in $Q(N, \Delta)$ as follows:

Subject to the constraints

$$
\begin{gathered}
p_{0} \geqq 0, \quad p_{1} \geqq 0, \quad \cdots, \quad p_{M} \geqq 0, \quad M=[N / 2], \\
p_{0}+2 \sum_{n=1}^{M} p_{n} \cos (2 \pi n k / N)=0, \quad k=\Delta+1, \Delta+2, \cdots, M, \\
p_{0}+2 \sum_{n=1}^{M} p_{n}=2 \pi,
\end{gathered}
$$

maximize the sum

$$
p_{0}^{2}+2 \sum_{n=1}^{M} p_{n}^{2}
$$

Since the constraints are linear, they describe the surface of a polyhedron in $(M+1)$-dimensional Euclidean space. The function we are trying to maximize on this surface has the property that, on any line segment, it assumes its maximum on one of the endpoints. (The square root of the function is a norm; the property follows from the triangle inequality.) Hence, on a polyhedron, it must assume its maximum on a vertex. The fundamental theorem of linear programming [2, p. 5] says that these vertices are the vectors $\left(p_{0}, \cdots, p_{M}\right)$ in the polyhedron which have at least $\Delta$ zerovalued coordinates. It turns out that specifying $\Delta$ zero-valued coordinates in a vector $\left(p_{0}, \cdots, p_{M}\right)$ in the polyhedron described by (2.4), (2.5), and (2.6) completely determines the vector.

One can see this by considering the rational function

$$
p(z)=(2 \pi / N) \sum_{k=-\Delta}^{\Delta} a(2 \pi k / N) z^{k}
$$


where $a$ is the function in $Q(N, \Delta)$ whose Fourier coefficients are $p_{0}, \cdots, p_{M}$. Since $a(2 \pi k / N)=a(-2 \pi k / N), p$ must be real-valued on the unit circle and symmetric in the sense that $p\left(e^{i x}\right)=p\left(e^{-i x}\right)$. In addition, since $p\left(e^{2 \pi i k / N}\right)=p_{k}, k=0,1, \cdots, M$, $p$ must be nonnegative at each of the roots of unity and take the value zero at $2 \Delta$ of them. All the roots are simple because the degree of $p$ indicates that it has only $2 \Delta$ roots. (The only exception to this is the case in which roots occur at +1 or -1 ; these must be double roots and the number of distinct roots of $p$ is diminished.) The rational function $p$ is determined, up to a constant factor, by its $2 \Delta$ roots. Thus, $\left(p_{0}, \cdots, p_{M}\right)$ is determined as soon as one chooses $\Delta$ of its coordinates to be zero.

Actually, we get a little more out of this argument: The zero coordinates of $\left(p_{0}, p_{1}, \cdots, p_{M}\right)$ must occur in adjacent pairs. This is so because $p\left(e^{i x}\right)$ must change sign at each of its simple zeroes, yet must remain positive at all the roots of unity. Therefore, if $N=2 M$ is even and $\Delta$ is odd, the set of zero coordinates of $\left(p_{0}, \cdots, p_{M}\right)$ can be written as $\left\{k_{0}, k_{1}, k_{1}+1, k_{2}, k_{2}+1, \cdots, k_{(\Delta-1) / 2}, k_{(\Delta-1) / 2}+1\right\}$ where $k_{0}=0$ or $k_{0}=M$ and $1 \leqq k_{j} \leqq M-2$ for $1 \leqq j \leqq(\Delta-1) / 2$. Similar statements can be made for the other possible values of $N$ and $\Delta$.

To compute the maximal function $a$ in $Q(N, \Delta)$, one needs only to compute the sum (2.7) for those vectors $\left(p_{0}, \cdots, p_{M}\right)$ in the polyhedron determined by placing $\Delta$ zeroes in adjacent pairs of coordinates. An elementary computation shows that

$$
p_{n}=\text { const } \prod_{i=1}^{\Delta} 4 \sin \left[\pi\left(n-m_{i}\right) / N\right] \sin \left[\pi\left(n+m_{i}\right) / N\right],
$$

where $\left\{m_{i}: 1 \leqq j \leqq \Delta\right\}$ is the set of zeroes in the coordinates of the vector $\left(p_{0}, \cdots, p_{M}\right)$. The value of const is determined by (2.6). The number of polyhedron vertices which must be searched is the binomial coefficient $C_{[N / 2]-\{\Delta / 2],\{\Delta / 2]}$. For values of $N$ smaller than 50, the computation of the maximal element in $Q(N, \Delta)$ can be made in less than a minute on a CDC 3600 computer.

One can compute the maximal element in the class $\operatorname{PL}(N, \Delta)$ by the same procedure, replacing the function to be maximized by

$$
p_{0}^{2}+2 \sum_{n=1}^{M} p_{n}^{2}\left(\frac{2}{3}+\frac{1}{3} \cos \frac{2 \pi n}{N}\right) \text {. }
$$

This is so because a straightforward computation shows that if $A \in \mathcal{P}(N, \Delta)$ and $\left\{p_{n}\right\}$ is the Fourier transform of the $Q(N, \Delta)$-function $a$ such that $a(2 \pi k / N)=$ $A(2 \pi k / N)$, then the integral $\int_{-\delta}^{\delta} A(x)^{2} d x$ is given by (2.8).

3. Results. The above algorithm was applied for a great many different values of $N$ and $\Delta$. In every case, the extremal function $A^{N, \Delta}$ in the class $P \mathcal{L}(N, \Delta)$ turned out to be unique and symmetric decreasing and there was an extremal function $a^{N, \Delta}$ in the class $Q(N, \Delta)$ such that $A^{N, \Delta}(2 \pi n / N)=a^{N, \Delta}(2 \pi n / N)$. The extremal function in $Q(N, \Delta)$ is not always unique because, when $N$ is even, $\left\{(-1)^{n}\right\}$ is a positivedefinite sequence of period $N$. Hence, if $a$ is maximal in $a(N, \Delta)$, then so is $a^{*}$, where $a^{*}(2 \pi n / N)=(-1)^{n} a(2 \pi n / N)$. However, nonuniqueness, in all cases computed, only occurred in this way.

In order to plot a graph of $D_{\delta}$, we computed $B(N, \Delta)$ and $Q(N, \Delta)$ for one hundred different values of $\delta=2 \pi(\Delta+1) / N$. Since we know that for $\delta$ of this form, $B(N, \Delta) \geqq$ $D_{\delta} \geqq Q Q Q(N, \Delta)$, we took $(B(N, \Delta)+Q(N, \Delta)) / 2$ as an estimate of $D_{\delta}$. The difference 
THE EXTREMAL CONSTANTS

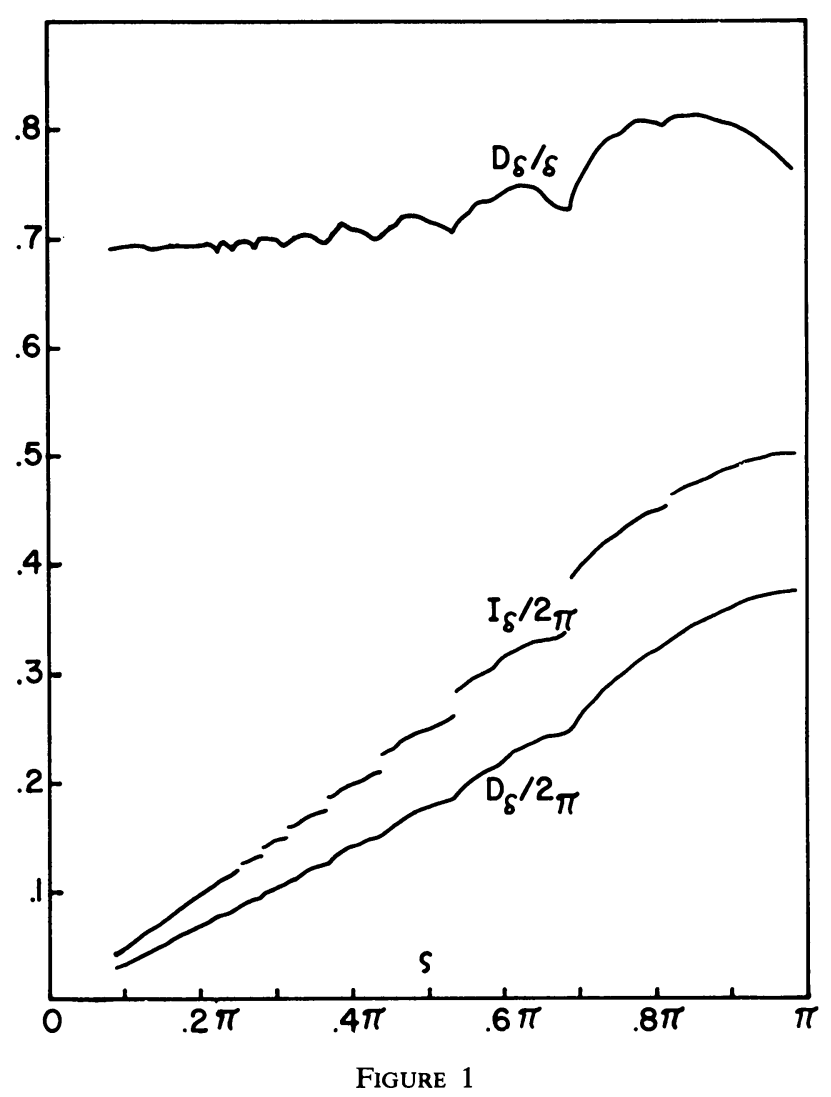

in the bounds on $D_{\delta}$ ranged between 1.6 percent and .01 percent of the estimated size of $D_{\delta}$. The difference tended to get smaller as $\delta$ got larger possibly because the number of nonzero mesh points of the extremal sequences was larger for large $\delta$, thus making the sequence a better approximation to the extremal function in $\mathfrak{C}_{\delta}$. A graph of the computed approximations to $D_{\delta}$ appears in Fig. 1 .

In the same manner, we plotted $D_{\delta} / \delta$ and the integral of the extremal function, which we denote by $I_{\delta}$. It is interesting that the value of $I_{\delta}$ seems to be independent of the manner in which $\delta=2 \pi(\Delta+1) / N$ is represented. That is, as long as the ratio $(\Delta+1) / N$ remains the same, the extremal function in the class $P \mathcal{L}(N, \Delta)$ has the same integral.

It appears that $I_{\delta}$ is discontinuous and $D_{\delta} / \delta$ is a sequence of humps. The endpoints of these humps correspond to the discontinuities in $I_{\delta}$. A possible explanation for this is that, at the points of discontinuity in $I_{\delta}$, the extremal function is not unique. This would give (at least) two possible values for $I_{\delta}$ at these points.

This explanation fits with the information given by the graphs of the extremal functions in the classes $\mathcal{P}(N, \Delta)$. For any particular $\delta=2 \pi(\Delta+1) / N$, the extremal function in $\operatorname{PL}(N, \Delta)$ takes on a characteristic shape, even for small values of $N$. It seems reasonable to consider its graph to be a reasonably accurate picture of the shape of an extremal function in the class $\mathcal{C}_{\delta}, \delta=2 \pi(\Delta+1) / N$. For convenience, we will call any such extremal function $\Lambda_{\delta}$. There appears to be some consistency 
OF EXTREMAL FUNCTIONS
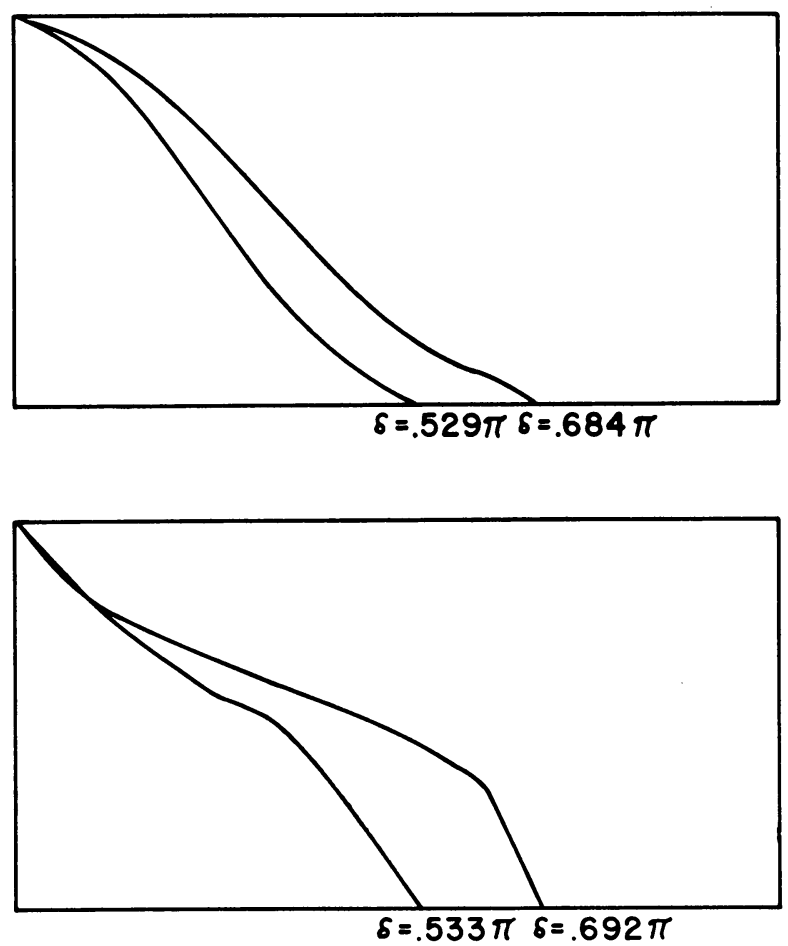

FIGURE 2

in the behavior of $\Lambda_{\delta}$ in the intervals between the points of discontinuity of $I_{\delta}$. As $\delta$ approaches a point of discontinuity of $I_{\delta}$ from the left, $\Lambda_{\delta}$ takes on a bell shape. When $\delta$ approaches such a point from the right, $\Lambda_{\delta}$ takes on a ski jump shape, as if the bell were reflected through the line from its top to its base. This phenomenon is illustrated in Fig. 2.

If the graphs of $\Lambda_{\delta}$ are all decreasing, which appears to be the case, then one can take a limit of the functions $\Lambda_{\delta}$ as $\delta$ approaches a point of discontinuity of $I_{\delta}$ through some sequence of values. Suppose $\delta_{n} \downarrow_{n} \delta_{0}$ and $\Lambda_{\delta_{n}} \rightarrow_{n} \Lambda^{(\mathrm{r})}$ pointwise, where $\delta_{0}$ is a point of discontinuity of $I_{\delta}$. Then, $\Lambda^{(\mathrm{r})} \in \mathcal{C}_{\delta_{0}}$ and

$$
D_{\delta_{0}} \geqq \int_{-\pi}^{\pi}\left|\Lambda^{(\mathrm{r})}(x)\right|^{2} d x=\lim _{n \rightarrow \infty} \int_{-\pi}^{\pi}\left|\Lambda_{\delta_{n}}(x)\right|^{2} d x=\lim _{n \rightarrow \infty} D_{\delta_{n}} \geqq D_{\delta_{0}} .
$$

Thus, $\Lambda^{(r)}$ is an extremal function in $\mathfrak{C}_{\delta_{0}}$.

Note that the existence of such a limit implies that $D_{\delta}$ is a right-continuous function of $\delta$. Suppose $D_{\delta}$ is also left-continuous and suppose $\delta_{n} \uparrow \delta_{0}$ and $\Lambda_{\delta_{n}} \rightarrow \Lambda^{(\ell)}$ pointwise. Then $\Lambda^{(\ell)} \in \mathfrak{C}_{\delta_{0}}$,

$$
D_{\delta_{0}} \geqq \int_{-\pi}^{\pi}\left|\Lambda^{(\ell)}(x)\right|^{2} d x=\lim _{n \rightarrow \infty} \int_{-\pi}^{\pi}\left|\Lambda_{\delta_{n}}(x)\right|^{2} d x=\lim _{n \rightarrow \infty} D_{\delta_{n}}=D_{\delta_{0}} .
$$

Thus, $\Lambda^{(\ell)}$ is also an extremal function in the class $\mathfrak{C}_{\delta_{0}}$.

The two extremal functions $\Lambda^{(r)}$ and $\Lambda^{(\ell)}$ should be different if the shape of the 
piecewise linear functions as $\delta$ nears $\delta_{0}$ is reasonably close to the shape of $\Lambda_{\delta}$. In particular, $\Lambda^{(\ell)}$ should have the bell shape and $\Lambda^{(r)}$ should have the ski jump shape.

Looking at the computational results, one notices an interesting pattern which makes it possible to guess the extremal function for values of $\delta$ which are rational multiples of $\pi$. If such a $\delta$ is expressed in the form $\delta=2 \pi(\Delta+1) / N$, with $N$ as small as possible, then the zeroes of the Fourier transform of the extremal function in $\operatorname{PS}\left(N^{\prime}, \Delta^{\prime}\right)$, where $\Delta^{\prime}$ and $N^{\prime}$ satisfy $\left(\Delta^{\prime}+1\right) / N^{\prime}=(\Delta+1) / N$, seem to have a repeated pattern in blocks of size $N$.

It is tempting to conjecture, from the observation on the periodicity of the zeroes of the Fourier transform, that if $\delta$ is a rational multiple of $2 \pi$, say $\delta=2 \pi p / q$ where $p$ and $q$ are integers with no common divisor, then the zeroes of the Fourier transform of the extremal function in $\mathfrak{C}_{\delta}$ must occur at the integers and must be periodic in the sense that if $n>0$ is a zero, then so is $n+q$.

In any case, by the Hadamard factorization theorem [1], the Fourier transform

$$
\mathcal{F} \Lambda_{\delta}(z)=\int_{-\delta}^{\delta} \Lambda(x) e^{-i z x} d x
$$

can be written in the form $e^{a z+b} \prod_{k=1}^{\infty}\left[1-\left(z / n_{k}\right)^{2}\right]$, where the $n_{k}$ are the zeroes of the entire function $\mathcal{F} \Lambda_{\delta}$.

Since $\mathcal{F} \Lambda_{\delta}(x)=\mathcal{F} \Lambda_{\delta}(-x)$, it must be that $a=0$. Therefore,

$$
\mathcal{F} \Lambda_{\delta}(z)=\text { const } \prod_{k=1}^{\infty}\left[1-\left(z / n_{k}\right)^{2}\right] \text {. }
$$

A theorem due to N. Wiener [5, p. 80] implies that $\mathcal{F} \Lambda_{\delta}$ is integrable over the real line, so that, at least in theory, one can invert the Fourier transform $\mathfrak{F} \Lambda_{\delta}$ to find $\Lambda_{\delta}$.

When $\delta=2 \pi(\Delta+1) / N$ and $N$ is not too large, one can guess the pattern of zeroes of $\mathcal{F} \Lambda_{\delta}$ by making computations using the algorithm described in the first part of this section. For example, in the case $\delta=\pi / 2$, the zero set of $\mathcal{F} \Lambda_{\pi / 2}$ appears to be $\{ \pm 4 k, \pm(4 k+1): 1 \leqq k<\infty\}$. The canonical product with these zeroes is

$$
\mathcal{F} \Lambda_{\pi / 2}(z)=\text { const }[\sin (\pi z / 4) / z] /[\Gamma(5 / 4+z / 4) \Gamma(5 / 4-z / 4)] \text {. }
$$

Fortunately, it is possible to explicitly invert this Fourier transform [4, p. 7]. One finds that

$$
\Lambda_{\pi / 2}(x)=\text { const } \chi * \alpha(x), \quad|x| \leqq \pi
$$

(the symbol $*$ denotes convolution), where

$$
\begin{aligned}
\chi & =1 \text { on }[-\pi / 4, \pi / 4] \\
& =0 \text { elsewhere, }
\end{aligned}
$$

and

$$
\alpha(t)=\chi(t)(\cos 2 t)^{1 / 2} .
$$

The square integral of this function, to fourteen decimal places, is 1.12504202380751. By computing the extremal elements in the classes $P \mathscr{L}(40,9)$ and $Q(40,9)$, one finds that $1.12213<D_{x / 2}<1.12785$. Thus, the square integral falls in the proper range. A graph of the function $\Lambda_{\pi / 2}$ is bell shaped, as one would expect from solutions to the discrete problem with $\delta$ near $\pi / 2$. 
Even though this evidence tends to verify the conjecture about the periodicity of the zeroes for the case $\delta=\pi / 2$, the author has an argument (not presented here) which shows that the conjecture cannot be true for some (very small) values of $\delta$.

Other cases, where the maximal function $\Lambda_{\delta}$ seems to have the form $\Lambda_{\delta}=$ const $\chi_{\delta} * \alpha_{\delta}$ where $\chi_{\delta}$ is the indicator function for the interval $[-\delta, \delta]$ and $\alpha_{\delta}(t)=$ $(\cos (\pi / \delta) t)^{\delta / \pi} \chi_{\delta}(t)$, occur when $\delta=2 \pi / n$ for $n=3,4,5, \cdots, 12$ (but not when $\delta=2 \pi / 13)$.

It may be worth noting that we can actually evaluate the constant involved when $\delta$ is of the form $\delta=2 \pi / n$. We require $\Lambda_{\delta}(0)=1$, hence

$$
\text { const }=1 / \int_{-\delta / 2}^{\delta / 2} \alpha_{\delta}(t) d t=1 / \mathfrak{F} \alpha_{\delta}(0) \text {. }
$$

Since [5, p. 7]

$$
\int_{-\delta}^{\delta} e^{i t s} /[\Gamma(1+1 / n+s / n) \Gamma(1+1 / n-s / n)] d s=n 2^{2 / n} \alpha_{2 \pi / n}(t) / \Gamma(1+2 / n),
$$

we have

$$
\text { const }=n 2^{2 / n} \Gamma(1+1 / n)^{2} / \Gamma(1+2 / n) \text {. }
$$

4. The Complex Case. If we enlarge the class $\mathcal{C}_{\delta}$ to include complex-valued functions, which is equivalent to removing the restriction that the Fourier coefficients be symmetric about zero, one can use the same techniques to find approximations to the maximal functions. All the arguments are essentially the same. (However, the fundamental theorem of linear programming must be slightly extended.)

Again, the cases $\delta=2 \pi / n$ turn out to be particularly nice. When $3 \leqq n \leqq 12$, the Fourier transform of the maximal functions appears to have its zeroes at the points $n k, n k+1$ where $k= \pm 1, \pm 2, \cdots$. It is easily verified that the periodic (period $2 \pi$ ), conjugate symmetric function $\Lambda_{\delta}$ such that

$$
\begin{aligned}
\Lambda_{\delta}(x) & =\left(e^{i x}-e^{i \delta}\right) /\left(1-e^{i \delta}\right), & & 0 \leqq x \leqq \delta, \\
& =0, & & \delta \leqq x \leqq \pi,
\end{aligned}
$$

is positive-definite when $\delta=2 \pi / n$, and its Fourier transform has its zeroes at the points $n k, n k+1, k= \pm 1, \pm 2, \cdots$. A straightforward computation shows that

$$
\int_{-\delta}^{\delta}\left|\Lambda_{\delta}(x)\right|^{2} d x=2(\delta-\sin \delta) /(1-\cos \delta) .
$$

For $\delta=\pi / 2$, the integral (4.1) is $\pi-2$, which fits with computational data giving upper and lower bounds on the maximum in this case.

5. Conclusion. The problems discussed in this paper are easy to state, yet very hard to solve analytically. Nevertheless, some analysis leads to a feasible computational approach. Results from the computations lead to some nice formulae for functions which, with some confidence, one can conjecture to be solutions.

6. Acknowledgment. I would like to thank Adriano Garsia for his help and encouragement during this work. 
Department of Mathematics and Computer Science

Colorado State University

Fort Collins, Colorado 80521

1. R. P. Boaz, Entire Functions, Academic Press, New York, 1954. MR 16, 914.

2. S. DAN $\varnothing$, Linear Programming in Industry. Theory and Applications: An Introduction, Springer-Verlag, Vienna, 1960. MR 24 \#B1667.

3. A. M. Garsia, E. Rodemich \& H. RuMSEY, "On some extremal positive definite functions," J. Math. Mech., v. 18, 1968/69, pp. 805-834. MR 40 \#4682.

4. W. MAGNUS \& F. OBERHETTINGER, Formeln und Sätze für die speziellen Funktionen der mathematischen Physik, Springer, Berlin, 1943; English transl., Chelsea, New York, 1949. MR 9, 183; MR 10, 532.

5. N. WIENER, The Fourier Integral and Certain of its Applications, Cambridge Univ. Press, Cambridge, 1933; reprint, Dover, New York, 1959. MR 20 \#6634.

6. A. ZYGMUND, Trigonometric Series. Vol. 1, 2nd ed., reprinted, Cambridge Univ. Press, New York, 1968. MR 38 \#488. 